\title{
Influence of Bactrian camel milk on the gut microbiota
}

\author{
Zhaoxia Wang, ${ }^{*} \dagger^{1}$ Wenyi Zhang, $\dagger^{1}$ Bini Wang, ${ }^{*}$ Fuxin Zhang, ${ }^{* 2}$ and Yuyu Shao*2 \\ ${ }^{*}$ College of Food Engineering and Nutritional Science, Shaanxi Normal University, Xi'an 710119, Shaanxi, P. R. China \\ †Key Laboratory of Dairy Biotechnology and Engineering, Ministry of Education, Inner Mongolia Agricultural University, Hohhot 010018 , \\ Inner Mongolia, P. R. China
}

\begin{abstract}
Bactrian camel milk has become popular in the market as an important source of nutrients with diverse functional effects. In this study, the influence of Bactrian camel milk on the gut microbiota of mice was studied using metagenomic-based sequencing of the V3 and V4 hypervariable regions of the $16 \mathrm{~S}$ rRNA gene. Bioinformatics analysis showed that Firmicutes and Bacteroidetes were the predominant phyla, accounting for more than $80 \%$ of the bacteria present. At the genus level, Allobaculum, Akkermansia, Romboutsia, Bifidobacterium, and Lactobacillus were most abundant in the gut microbiota; of these, Allobaculum and Akkermansia were the predominant genera, representing 40.42 and $7.85 \%$ of all the bacteria present, respectively. Camel milk was found to reduce relative abundance of Romboutsia, Lactobacillus, Turicibacter, and Desulfovibrio (decreased by 50.88, 34.78, 26.67, and $54.55 \%$, respectively) in the gut microbiota compared with the control. However, some genera such as Allobaculum, Akkermansia, and Bifidobacterium in the gastrointestinal flora increased in abundance in the presence of camel milk; these genera are correlated with beneficial effects for organisms. Our research suggests that the gut microbiota should be taken into account when conducting functional studies on camel milk, and this work provides a useful foundation for further study on functions of camel milk.
\end{abstract}

Key words: camel milk, gut microbiome, probiotic, high-throughput sequencing

\section{INTRODUCTION}

Camels are important nonbovine animals that produce milk rich in nutrients for human consumption (Lajnaf et al., 2017). Two species belonging to the $\mathrm{Ca}$ -

\footnotetext{
Received September 18, 2017.

Accepted March 18, 2018.

${ }^{1}$ These authors contributed equally to this work.

${ }^{2}$ Corresponding authors: fuxinzh@snnu.edu.cn and icheesemaking@ yahoo.com
}

melidae family include Bactrian camels with 2 humps (Camelus bactrianus) and Dromedary camels with a single hump (Camelus dromedarius; Cui et al., 2007). The studies estimated a total population of 22 million camels in the world, of which $89 \%$ were $C$. dromedarius located in North Africa and West Asia and the remaining $11 \%$ were C. bactrianus distributed mainly in central Asian countries, including China and Mongolia (Silbermayr et al., 2010; Mihic et al., 2016). China has only C. bactrianus, which is mainly distributed in the desert and grasslands of Xinjiang (55\%) and Inner Mongolia (41\%). There are 3 breeds within the species; namely, the Xinjiang camel, the Alxa camel, and the Sonid camel, named according to the geographic area in which they are found (Sa et al., 2015). Commercial Bactrian camel milk can be found in local markets and has become popular in China in recent years.

Although the numbers of $C$. bactrianus are relatively low compared with $C$. dromedarius, the nutrients in the milk of Bactrian camels are higher in protein, DM, and fat and lower in lactose than milk from Dromedary camels (Konuspayeva et al., 2009). Studies on the functions of camel milk have shown that it has good properties for human health, including prevention of diabetes, cancer, immune disorders, allergic symptoms, Crohn's disease, hypertension, oxidative stress, lipid peroxidation, and autism (Yadav et al., 2015; Kaskous, 2016). It has high levels of MUFA and PUFA, vitamin C, lactoferrin, immunoglobulins, serum albumin, lysozyme, insulin, iron, and manganese and low levels of $\alpha-\mathrm{CN}$ and $\beta$-LG (Brezovečki et al., 2015; Kaskous, 2016).

Interplay among food, disease, and the gut microbiota has been studied in recent years (Dolan and Chang, 2017; Espín, 2017). Several studies have shown that certain foods can modulate the species composition and community structure of the gut microbiota due to changes in the ecological environment in the gut (e.g., bile acids and $\mathrm{pH}$ ) and that different nutrients in foods can be selectively used by different microbes (McKenzie et al., 2017). The gut microbiota can be changed, even within a day, when the diet is changed (Koropatkin et al., 2012). Meanwhile, species composition of the gut microbiota can be different in individuals with various 
diseases compared with healthy individuals (Cani et al., 2016). Reports have indicated correlations between gut microbiota and obesity, diabetes, inflammatory bowel disease, and cancer; in particular, changes in the quantity of some microbial genera could either induce certain diseases or provide health benefits (Cani et al., 2016; Erdman, 2016; Knip and Siljander, 2016; Miyoshi and Chang, 2017). Comparative studies led us to conclude that although there are abundant nutrients in foods that have beneficial functional effects on human health, we cannot neglect the fact that these functional studies should not be independent of the gut microbiota. Therefore, when we studied the function of camel milk, its influence on microbiota should be investigated to comprehensively understand its function. In this research, the V3 and V4 hypervariable regions of $16 \mathrm{~S}$ rRNA gene amplicon sequencing was used to investigate the effects of camel milk on the gut microbiota to provide a fundamental basis for functional studies on camel milk.

\section{MATERIALS AND METHODS}

\section{Collection of Gut Microbiota Samples}

Twelve-week-old C57BL/6J male mice were housed with 12-h light-dark cycles at a temperature of $22 \pm$ $2^{\circ} \mathrm{C}$ and a humidity of $45 \pm 5 \%$ and fed sterilized standard food and distilled water ad libitum. The animals received humane care, and all procedures involving them were performed in accordance with institutional guidelines.

After acclimation for $1 \mathrm{wk}$, the mice were allocated randomly to 2 groups ( $\mathrm{n}=6 \mathrm{mice} /$ group): mice that received $10 \mathrm{~mL}$ of sterile distilled water $/ \mathrm{kg}$ of $\mathrm{BW}$ intragastrically (DW) and mice that received $10 \mathrm{~mL}$ of camel milk $/ \mathrm{kg}$ of BW intragastrically (CM; Arab et al., 2017). Each group was caged individually (1 mouse/cage) to avoid any direct contact between animals. Commercial UHT Bactrian camel milk, which had a 6-mo shelf life, was purchased from the market and stored at $4^{\circ} \mathrm{C}$; the same batch of UHT camel milk was used for the entire duration of the experiment. All groups of mice were treated once a day for 4 consecutive weeks. Fecal samples were collected on d 29 and placed in liquid nitrogen and stored at $-80^{\circ} \mathrm{C}$ before metagenomic DNA extraction.

\section{Metagenomic DNA Extraction}

Metagenomic DNA from the microbiome present in fecal samples was extracted and analyzed. For extraction, we used the commercial kit (QIAamp DNA Stool Mini Kit; Qiagen, Valencia, CA) according to the manufacturer's instructions. The concentration and purity of the metagenomic DNA were evaluated using a spectrophotometer (NanoDrop 2000; Thermo Fisher Scientific, Waltham, MA); the quality of the metagenomic DNA was assessed by $1 \%$ agarose gel electrophoresis at a voltage of $100 \mathrm{~V}$ for $40 \mathrm{~min}$. High-quality DNA was diluted to $1 \mathrm{ng} / \mu \mathrm{L}$ in sterile water as the template for PCR.

\section{High-Throughput Sequencing of V3-V4 Regions of 16S rRNA Gene}

Amplification of the V3-V4 regions of the 16S rRNA gene was achieved using specific primers with a set of 12-nucleotide barcodes (Table 1). We used the universal forward primer 338F (5'-ACTCCTACGGGAGGCAGCA-3') and the reverse primer 806R (5'-GGACTACHVGGGTWTCTAAT-3') for PCR, which was done with Phusion High-Fidelity PCR Master Mix (Thermo Fisher Scientific Inc.; Zhang et al., 2017). Amplified PCR products were detected by electrophoresis in $2 \%$ agarose gels at a voltage of $80 \mathrm{~V}$ for $40 \mathrm{~min}$. The PCR products were purified using the Qiagen Gel Extraction Kit (Qiagen Inc., Germantown, MD). A TruSeq DNA PCR-Free Sample Preparation Kit (Illumina Inc., San Diego, CA) was used to construct the DNA library. The library was quantified with a Qubit fluorometer (Thermo Fisher Scientific) and an Agilent 2100 Bioanalyzer system (Agilent Technologies, Santa Clara, CA). The sequencing was done using an Illumina HiSeq 2500 system (Illumina Inc.), and 250-bp paired-end reads were generated.

\section{Bioinformatics Analysis of the Sequence Data}

Paired-end reads from different samples were separated based on barcode sequences. Flash (v. 1.2.7;

Table 1. Amplicon sequencing information of the gut microbiota from the camel milk (CM) group and the distilled water (DW) group of mice

\begin{tabular}{llcc}
\hline Sample & Barcode sequence & $\begin{array}{c}\text { Effective } \\
\text { tags (no.) }\end{array}$ & Q20 \\
\hline CM1 & GATCAG, ACTGAT & 55,457 & 97.25 \\
CM2 & TAGCTT, ACTGAT & 51,562 & 97.33 \\
CM3 & GGCTAC, ACTGAT & 51,241 & 97.35 \\
CM4 & CTTGTA, ACTGAT & 56,028 & 97.33 \\
CM5 & ATCACG, ATGAGC & 56,107 & 97.26 \\
CM6 & CGATGT, ATGAGC & 54,278 & 97.25 \\
DW1 & ATCACG, ACTGAT & 52,918 & 97.32 \\
DW2 & CGATGT, ACTGAT & 50,338 & 97.36 \\
DW3 & TGACCA, ACTGAT & 53,306 & 97.25 \\
DW4 & ACAGTG, ACTGAT & 52,146 & 97.39 \\
DW5 & GCCAAT, ACTGAT & 54,978 & 97.24 \\
DW6 & ACTTGA, ACTGAT & 56,317 & 97.50 \\
\hline
\end{tabular}

${ }^{1} \mathrm{Q} 20$ : The bases with minimum base call accuracy of $99 \%$ in effective tags. 
http://ccb.jhu.edu/software/FLASH/) was used to merge the paired-end reads with barcode and primer free to obtain raw tags. Qiime pipeline (v. 1.7.0; http:// qiime.org/scripts/split_libraries_fastq.html) was used to filter out low-quality tags. Clean tags were compared with the Gold database using the UCHIME algorithm to detect and remove chimera sequences and obtain effective tags for further analysis (Haas et al., 2011).

Uparse software (v. 7.0.1001; http://drive5.com/ uparse/) was used to cluster effective tags to the operational taxonomic units (OTU) based on 97\% similarity of sequences (Zhang et al., 2017). Representative OTU with high frequency of occurrence were selected and annotated for taxonomic information using the Mothur method and SSUrRNA database in SILVA (http:// www.arb-silva.de/) with a threshold of 0.8 to 1 (Quast et al., 2013) to obtain community compositions at different taxonomic levels (phylum, class, order, family, genus, and species). Multiple sequence alignments were performed using Muscle software to study phylogenetic relationships among different OTU and the predominant bacteria (at different taxonomic level) in gut microbiota. Normalization of the OTU abundance was achieved using the standard sequence number corresponding to the sample with the least number of sequences. Alpha diversity and beta diversity were analyzed based on these normalized data.
Indices of Chao1, Simpson, Shannon, abundancebased coverage estimator, and observed species were calculated by Qiime to study alpha diversity. Shannon and Simpson were used for measurement of the microbial community diversity, and abundance-based coverage estimator, Chao1, and observed species were used for measurement of microbial community richness (De Nardi et al., 2016; Ji et al., 2017). Weighted and unweighted UniFrac distances between microbial communities from fecal samples were calculated by Qiime to study beta diversity metrics (Galloway-Peña et al., 2017). The Anosim and MRPP functions in the vegan package of R software (v. 2.15.3; https://www.r-project .org/) were used to conduct the Anosim and MRPP analysis. Analysis of differences between 2 groups was performed by Wilcoxon test in $\mathrm{R}$ software. Taxonomic and phylogenetic trees of the gut microbiota were visualized using Graphlan software (https://bitbucket.org/ nsegata/graphlan/src). R software was used for plotting and the statistical analysis in this study.

\section{RESULTS}

\section{High-Throughput Sequencing Information for the Gut Microbiota}

Amplicons of the V3-V4 hypervariable regions of the $16 \mathrm{~S}$ rRNA gene were sequenced with Illumina

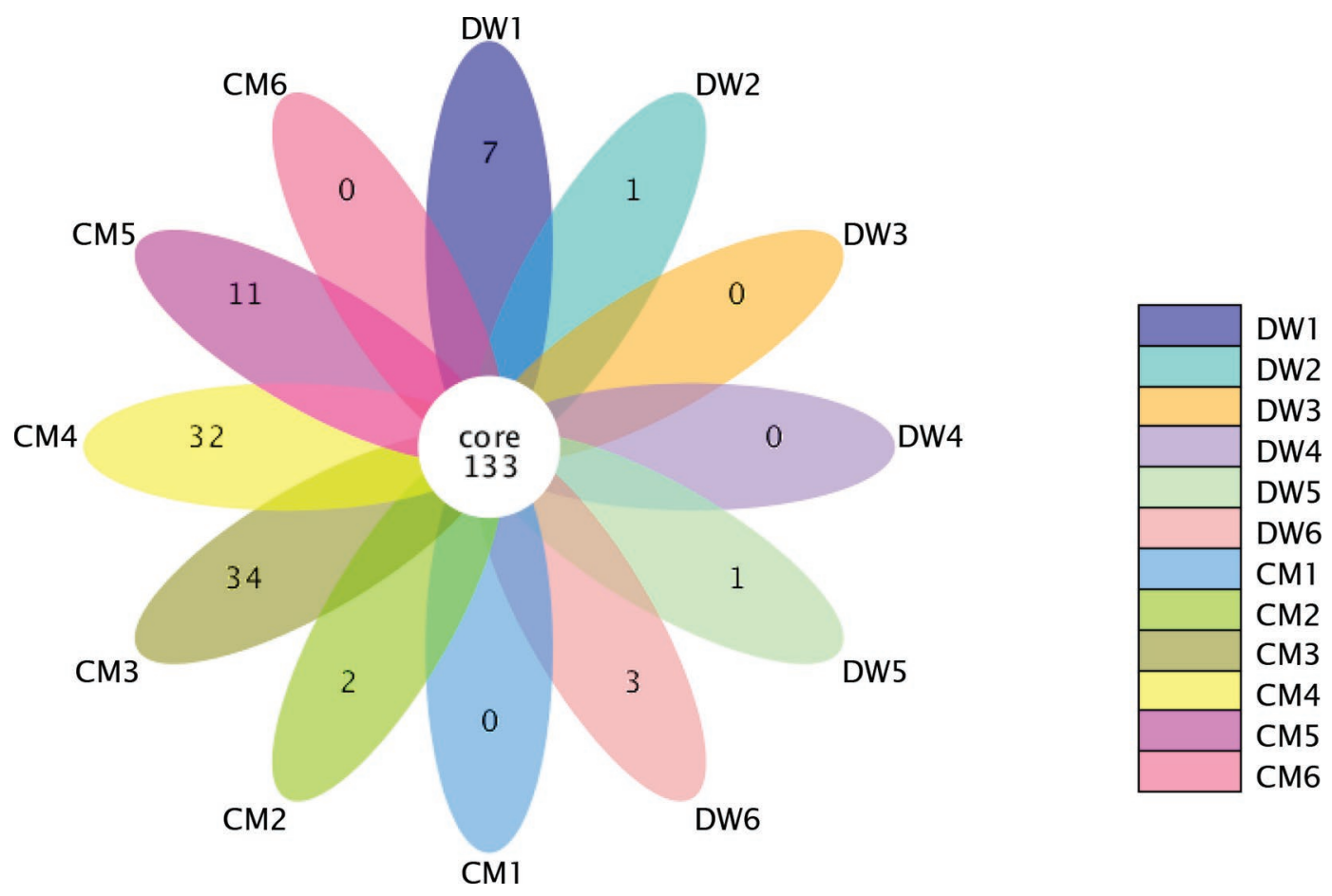

Figure 1. Number of operational taxonomic units of the gut microbiota from the camel milk (CM) group and the distilled water (DW) group of mice. Color version available online. 


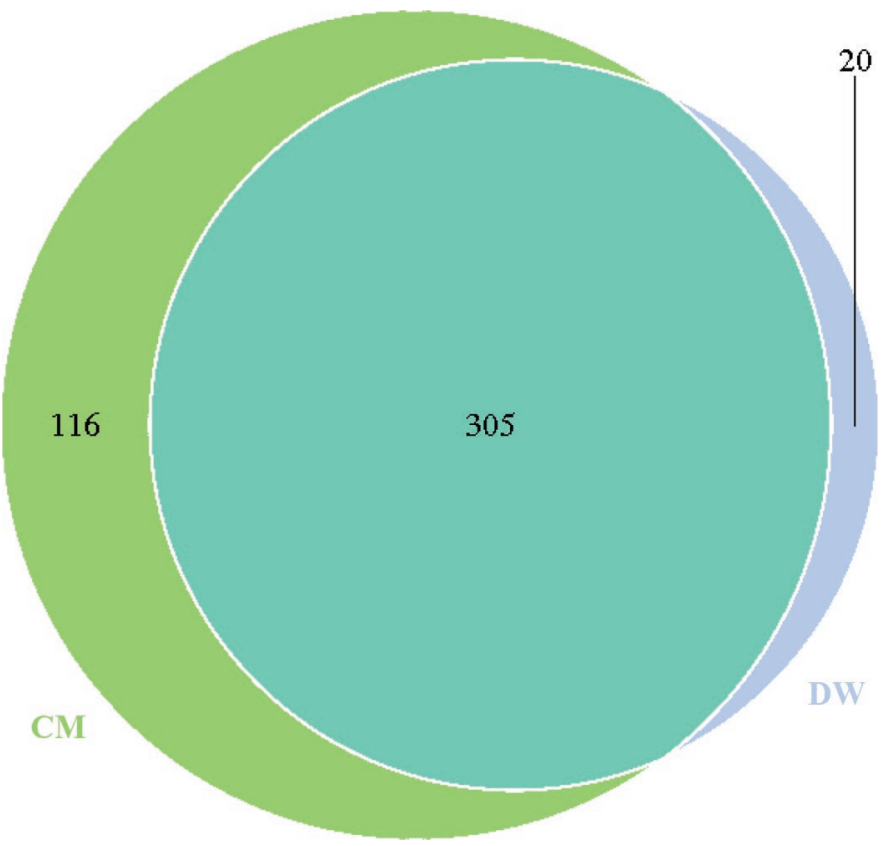

Figure 2. Venn graph of operational taxonomic units of the gut microbiota from the camel milk (CM) group and the distilled water (DW) group of mice. Color version available online.

HiSeq2500, obtaining a total of 644,676 high-quality sequences (i.e., effective tags), with an average of 53,723 sequences for each of the 12 samples (Table 1). The bases with minimum base call accuracy of $99 \%$ in effective tags (Q20) for each amplicon sequenced were all higher than $97.24 \%$, indicating high-quality sequencing data suitable for further study.
Table 2. The main bacterial phyla and genera in the gut microbiota from the camel milk (CM) group and the distilled water (DW) group of mice

\begin{tabular}{llcc}
\hline & & \multicolumn{2}{c}{ Mean proportion } \\
\cline { 3 - 4 } Taxonomic & & $\mathrm{CM}$ & $\mathrm{DW}$ \\
\hline level & Bacterial taxonomy & 0.621 & 0.618 \\
& Firmicutes & 0.173 & 0.226 \\
& Bacteroidetes & 0.094 & 0.063 \\
Verrucomicrobia & 0.094 & 0.071 \\
& Actinobacteria & 0.013 & 0.015 \\
Proteobacteria & 0.441 & 0.367 \\
& Allobaculum & 0.094 & 0.063 \\
& Akkermansia & 0.028 & 0.057 \\
& Romboutsia & 0.080 & 0.059 \\
& Bifidobacterium & 0.030 & 0.046 \\
& Lactobacillus & 0.011 & 0.015 \\
& Turicibacter & 0.005 & 0.011 \\
& Desulfovibrio & 0.003 & 0.004 \\
& Pseudomonas & 0.004 & 0.007 \\
& Lachnoclostridium & 0.006 & 0.009 \\
& Alistipes & 0.004 & 0.004 \\
& Odoribacter & 0.002 & 0.003 \\
\hline Bacteroides & & \\
& &
\end{tabular}

\section{OTU Analysis and Taxonomic Annotation of the Gut Microbiota from the CM Group and DW Group of Mice}

There were 133 mutual OTU (core OTU) in each of the 12 gut microbiota samples (Figure 1). When the 12 samples were allocated to DW and CM groups, 305 mutual OTU were detected in the gut microbiota from the 2 groups of mice (Figure 2).

Phylum and genus information is shown in Table 2 and Figure 3. The results showed that Firmicutes, Bacteroidetes, Verrucomicrobia, Actinobacteria, Pro-

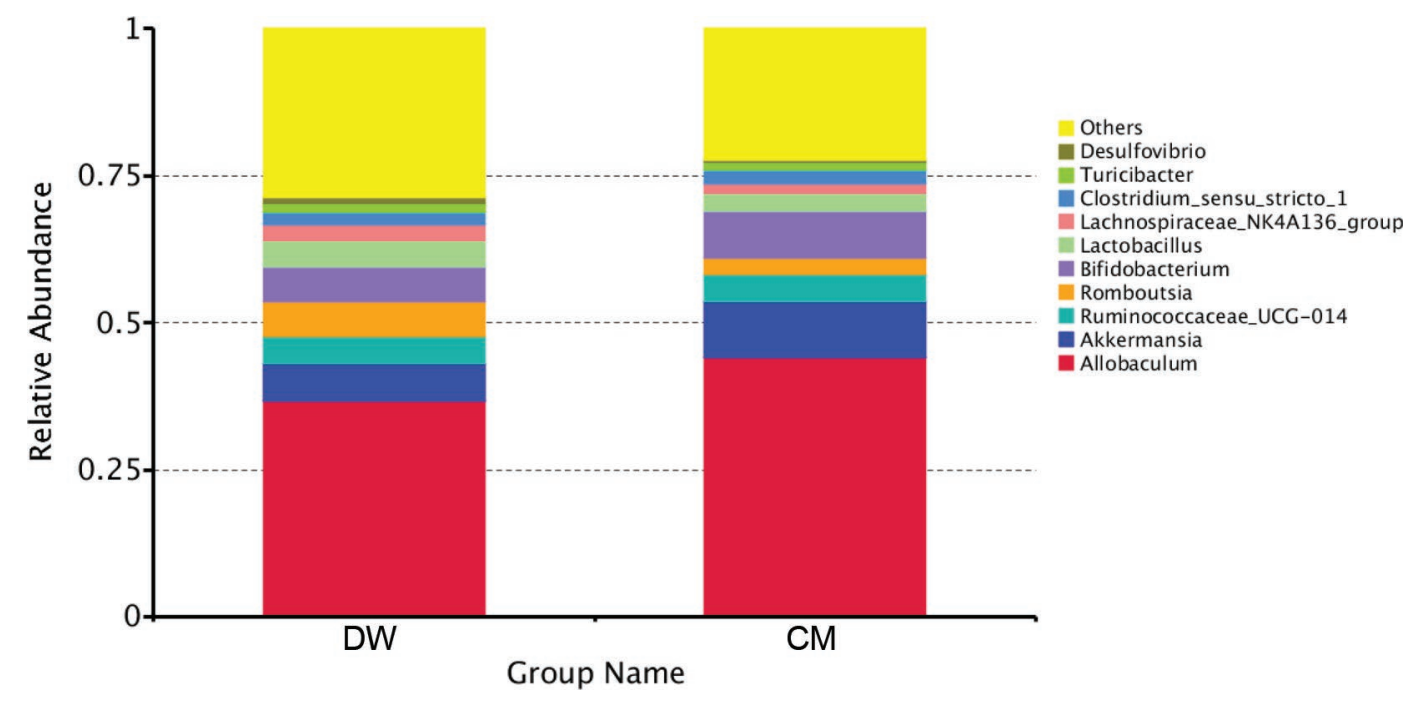

Figure 3. Relative abundance of different bacterial genera in the gut microbiota from the camel milk (CM) group and the distilled water (DW) group of mice. Color version available online. 
teobacteria, and Saccharibacteria were the main phyla present in the microbiota, of which Firmicutes and Bacteroidetes represented more than $80 \%$ of the bacteria present and were therefore the predominant phyla. At the genus level, Allobaculum, Akkermansia, Rombout- sia, Bifidobacterium, Lactobacillus, Turicibacter, and Desulfovibrio were the main genera detected in the gut microbiota. Allobaculum and Akkermansia were the predominant genera, representing 40.42 and $7.85 \%$ of the bacteria in the gut microbiota samples, respec-

\section{(a)}

A:c--Bacteroidia

B:---Bacteroidales
C:f--Rikenellaceae

E:o---Bifidobacteriales

:-Bifidobacterium

H:C--Coriobacteriia

:o---Coriobacteriales

K: --Coriobacteriaceae UCG-002

L:C--Bacilli

N:f--Lactobacillaceae

O:g--Lactobacillus

Q:f--Lachnospiracea

R:c--Clostridia

S:0--Clostridiales

$\mathrm{U}: \mathrm{g}-$-Clostridium sensu stricto

W:g--Romboutsia

X:f--Ruminococcacea

Y:f-Lachnospiraceae

Z:c--Erysipelotrichia

b:f--Erysipelotrichaceae

c:g-Turicibacter

d:c--Gammaproteobacteri

e:o---Pseudomonadales

g:o--Verrucomicrobiales

h:f--Verrucomicrobiaceae

i:g--Akkermansia

\section{OTU Tree of CM by GraPhIAn}

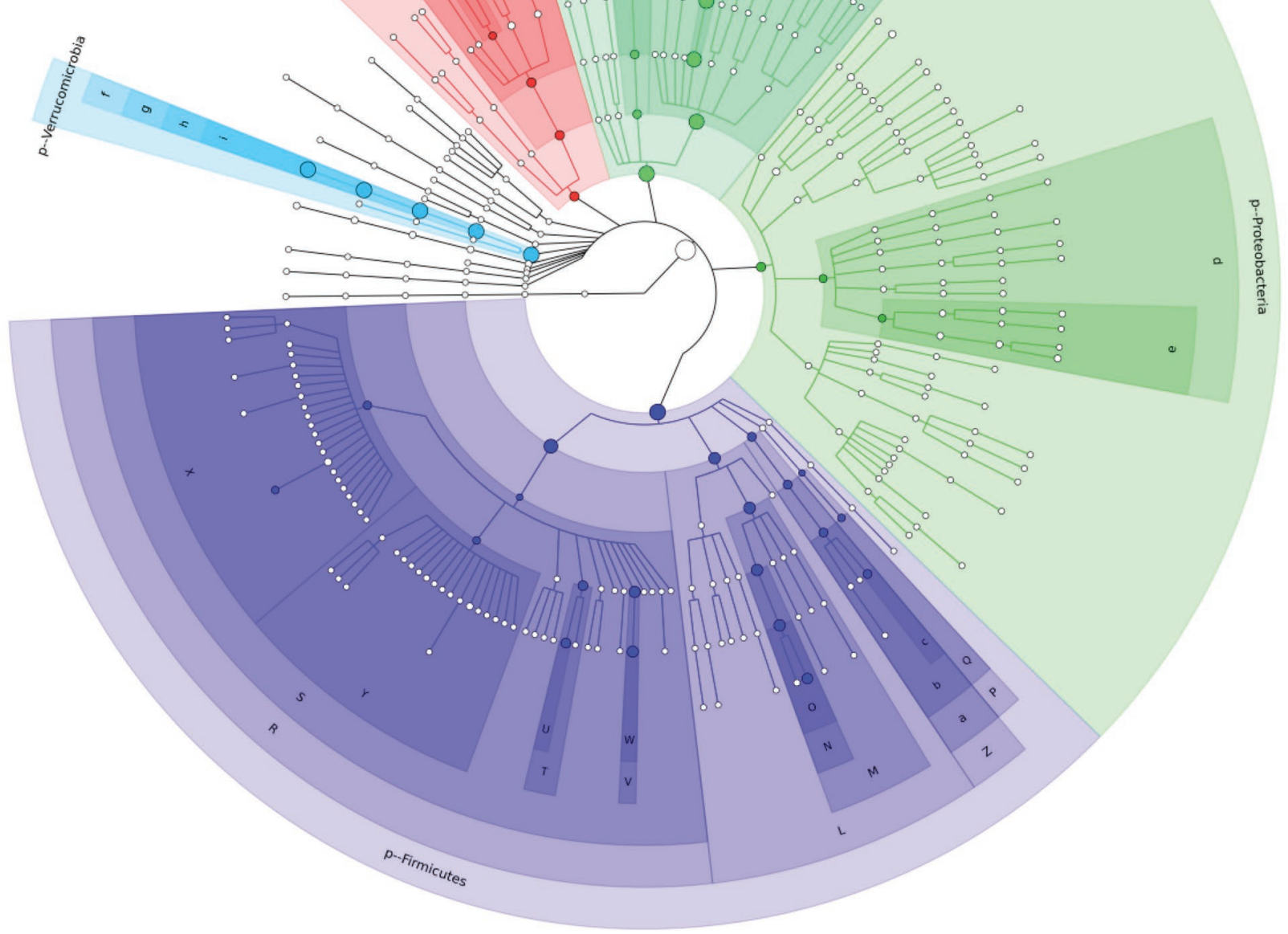

Figure 4. Taxonomic and phylogenetic trees of the gut microbiota from the camel milk (CM) group (a) and the distilled water (DW) group (b) of mice. OTU = operational taxonomic units. Color version available online. 
tively. The taxonomic and phylogenetic trees of the gut microbiota from the CM group and the DW group of mice are presented to compare bacterial taxonomy at different phyla and genera levels in the different gut microbiota samples (Figures 4 and 5).

\section{Alpha Diversity and Beta Diversity of the Gut Microbiota}

Species accumulation was analyzed to investigate whether the sample size is sufficient to evaluate mi-

\section{(b)}

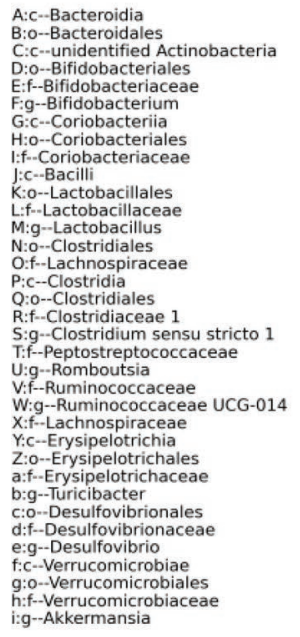

OTU Tree of DW by GraPhIAn

P-ACTINOBACTERIA P--BACTEROIDETES
P-FIRMICUTES

P--FIRMICUTES
P.-PROTEOBACTERIA

D: o- Bifidobacteriales

P.PROTEOBACTERIA

f--Lactobacillales

M:g--Lactobacillus

:f--Lachnospirace

Q:--Clostridiales

: g-Clostridium sensu stricto I

U: g--Romboutsia

W:g--Ruminococcaceae UCG-014

Y:- - Erysipelotrichia

2.--Erysipelotrichales

:---uricibacter d:f-Desulfovibrionacea :--Verrucomicrobia

h:f--Verrucomicrobiacea
g--Akkermansia
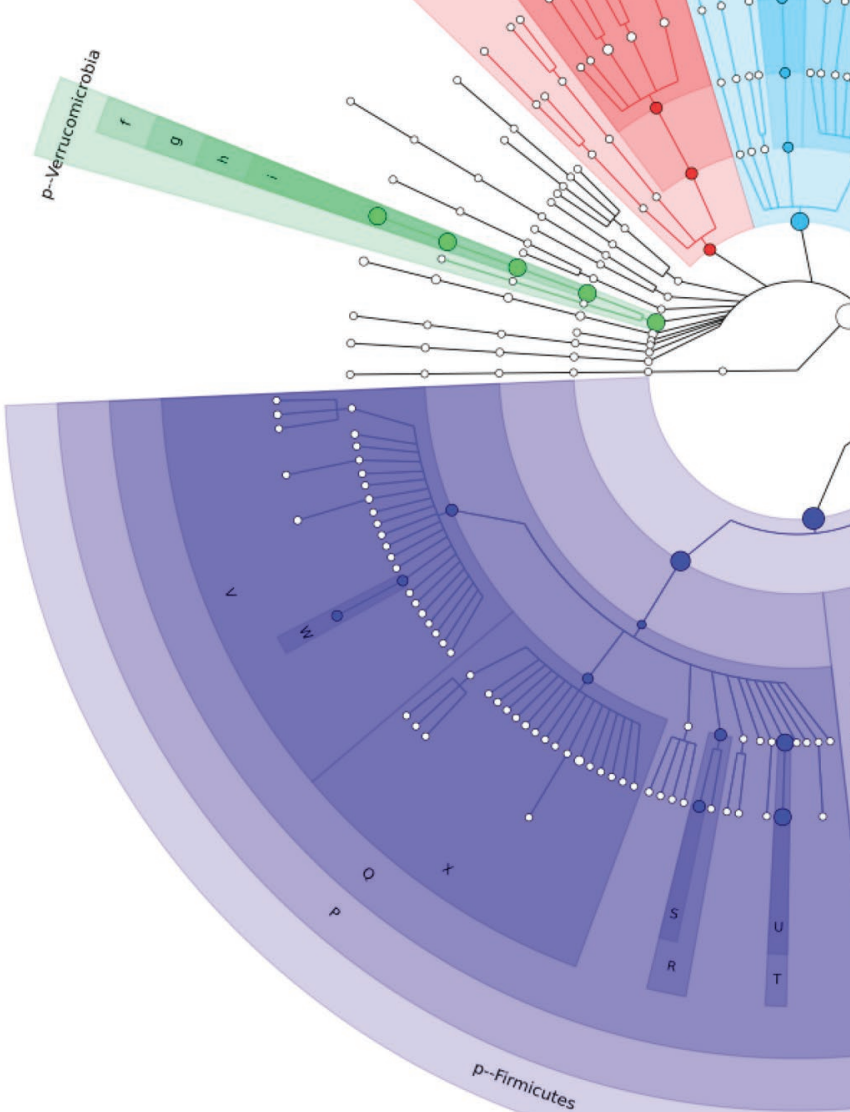

Figure 4 (Continued). Taxonomic and phylogenetic trees of the gut microbiota from the camel milk (CM) group (a) and the distilled water (DW) group (b) of mice. OTU = operational taxonomic units. Color version available online. 
crobial diversity. The result is presented in continuous boxplots (Figure 6) showing that the environmental species tended to be stable when sample size increased to 12 , indicating that the sample size is sufficient for further study. Alpha diversity was analyzed to measure diversity of the microbial community within a sample (Ai et al., 2017). The results showed that alpha diver- sity was higher in the gut microbiota from the CM group of mice than from the DW group of mice (Table 3 ), indicating that the richness and evenness of the gut microbiota had increased following administration of camel milk; however, the difference in alpha diversity between gut microbiota of the 2 groups of samples was not statistically significant $(P>0.05)$.

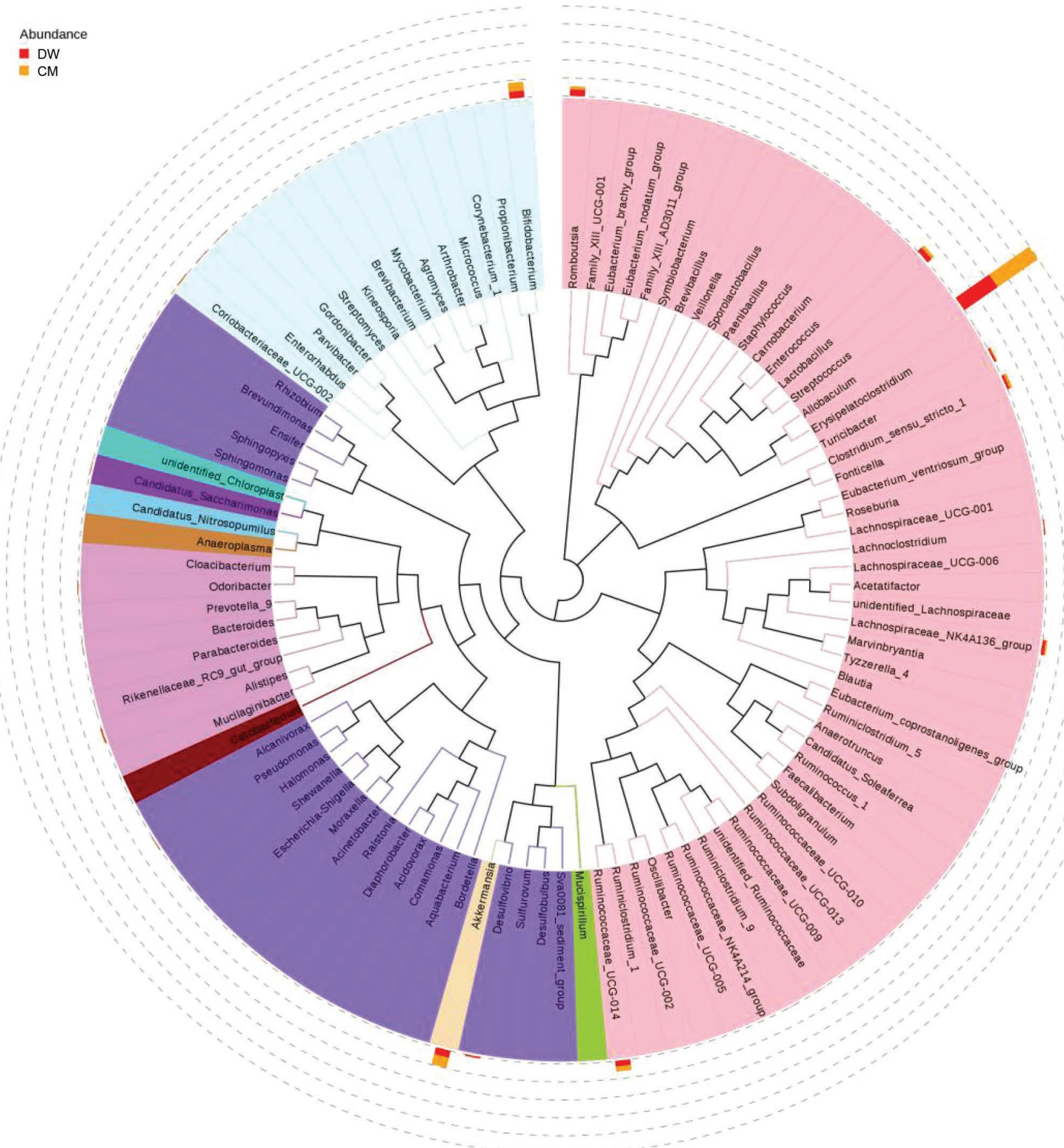

Figure 5. Phylogenetic evolutionary tree of the gut microbiota from the camel milk (CM) group and the distilled water (DW) group of mice at the genus level. Color version available online. 
Beta diversity is a comparative analysis of microbial community composition between each sample pair in complexity (Yan et al., 2016). The beta diversity between the 2 groups of gut microbiota is presented in Figure 7. Principal coordinate analysis based on weighted UniFrac distance showed that microbial community of the 2 groups of gut microbiota is distributed separately (Figure 8). There were significant differences in beta diversity between the 2 groups of gut microbiota based on the unweighted UniFrac $(P=0.0066)$ and weighted UniFrac $(P=0.0235)$ distances. Anosim analysis also showed that there were significant differences $(\mathrm{R}=$ $0.3352, P=0.007$; Figure 9) in gut microbiota between the CM group and the DW group of mice. This result was similar to the MRPP analysis, which indicated that differences between groups were higher than the differences within each group $(\mathrm{A}=0.0816, P=0.004)$.

\section{DISCUSSION}

In this study, we investigated the influence of camel milk on gut microbiota in the mouse model. The results of alpha diversity and beta diversity analyses suggest that there is a correlation between camel milk and changes in the gut microbiota. Although the differences of the alpha diversity between the gut microbiota of the CM groups and the DW groups were insignificant, alpha diversity increased when mice were fed camel milk. Beta diversity analysis did show significant differences between the 2 groups in their gut microbiota. Relatively, there was a higher abundance of Allobaculum, Akkermansia, and Bifidobacterium in gut microbiota of the mice fed camel milk, indicating that camel milk can enhance and improve the environment for proliferation of these genera.

Allobaculum has been reported to have potential beneficial effects on the host (Tachon et al., 2013). Zhang et al. (2012) studied the Chinese herb berberine, which is used for treatment of bacterial diarrhea and the prevention of obesity and insulin resistance in rats fed high-fat diets; they found that increases in the abundance of Allobaculum were found in rats treated with berberine, which may increase production of shortchain fatty acids (SCFA), whereas a lower abundance

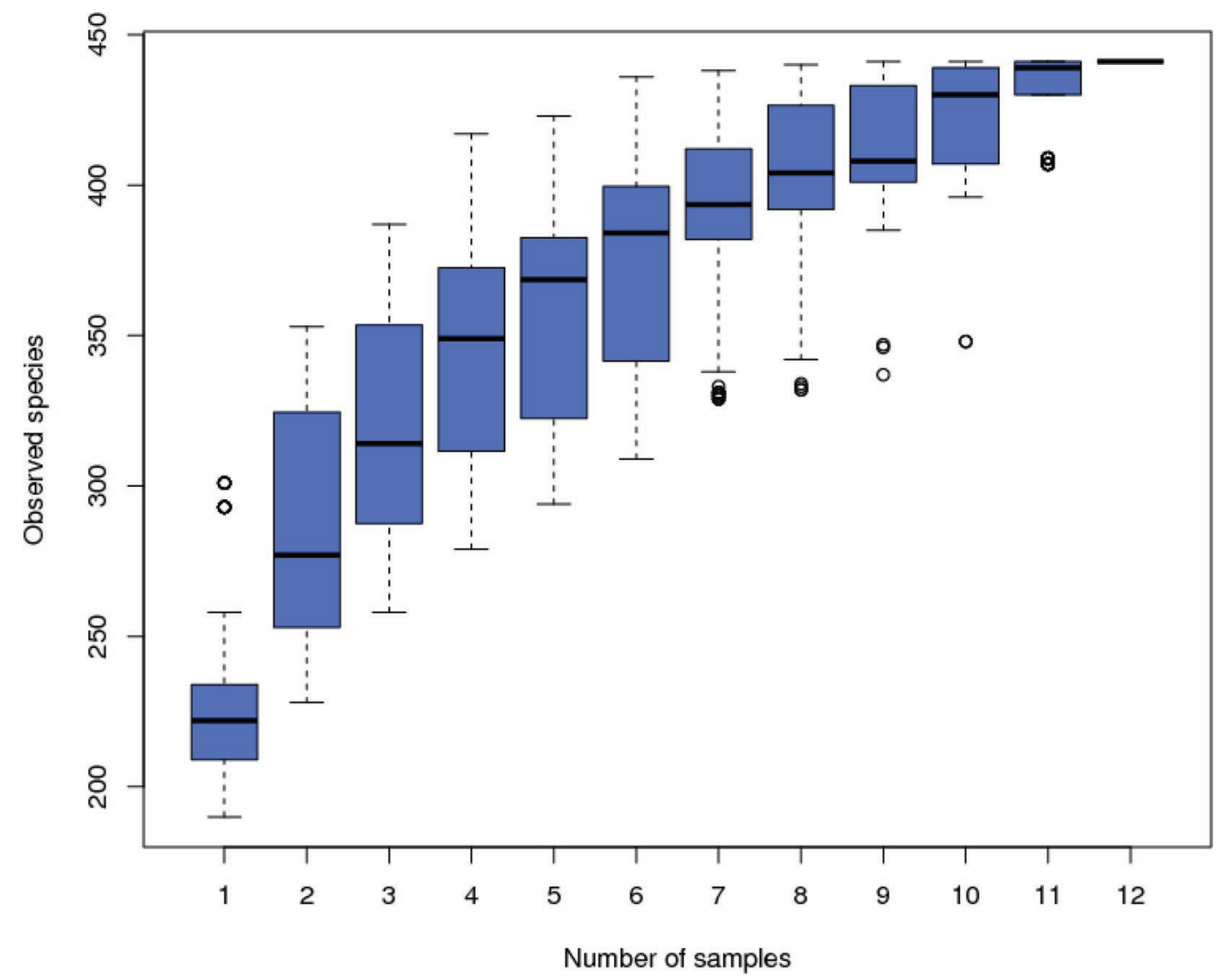

Figure 6. Estimation of the sample size by species accumulation analysis. The limits of the box (the bottom and top of the box) are the first and third quartiles of the number of operational taxonomic units (OTU), and the line (band inside the box) is the second quartile of the number of OTU (the median). The ends of the whiskers represent the minimum and maximum of the number of OTU. The out-of-whiskers outliers are presented with the dots. Color version available online. 
of Allobaculum was recorded in the control rats fed only the high-fat diet. This study indicated that the genus Allobaculum may have an intimate relationship with obesity and could be considered an indicator bacterial genus for obesity. Genera that produce SCFA, such as Allobaculum and Bifidobacterium, could have beneficial effects on an organism through the functions of SCFA, which include properties associated with colon health and anti-inflammation. Similar research has shown that the genus Allobaculum was negatively correlated with adiposity, which increased in C57BL/6 mice fed a lowfat diet compared with mice fed a high-fat diet (Baldwin et al., 2016). Our study indicates that camel milk could enhance the abundance of Allobaculum, which may positively influence the physiological function of the organism.

Akkermansia is a mucin-degrading probiotic that is well known for its positive effects on diabetes, obesity, metabolic disorders, and inflammation (Belzer and Vos, 2012). Abundance of this genus can be reduced or increased by dietary mediation. The beneficial effects of Akkermansia on obesity and diabetes are mainly due to their positive modulation of the mucus thickness and gut barrier integrity, which can be disrupted by high-fat diets (Shang et al., 2017). Foods containing fiber, prebiotics, and other probiotics could increase the abundance of Akkermansia (Krumbeck et al., 2016; Morowitz et al., 2017), whereas high-fat diets can decrease the abundance of this genus (Grander et al., 2018). Previous studies have focused on the therapeutic effects of camel milk on diabetes, particularly in relation to the composition of camel milk (Malik et al., 2012); this includes insulin-like proteins and their relationship with blood glucose levels as well as related indices of low-density lipoprotein, high-density lipoprotein, cholesterol, triacylglycerols, and enzymes (catalase, glutathione, superoxide dismutase; Mihic et al., 2016). Only a few studies have considered the effect of camel milk on the gut microbiota and subsequent influences on associated diseases. Our study found that camel milk could increase the abundance of Akkermansia, which can have a positive effect on associated diseases. Con-

Table 3. Alpha diversity of the gut microbiota from the camel milk (CM) group and the distilled water (DW) group of mice

\begin{tabular}{lrr}
\hline \multirow{2}{*}{ Sample } & \multicolumn{2}{c}{ Mean } \\
\cline { 2 - 3 } & \multicolumn{1}{c}{ CM } & \multicolumn{1}{c}{ DW } \\
\hline Abundance-based coverage estimator & 262.99 & 239.02 \\
Chao1 & 261.09 & 235.70 \\
Observed species & 244.00 & 227.00 \\
Shannon & 4.20 & 4.60 \\
Simpson & 0.85 & 0.90 \\
\hline
\end{tabular}

sequently, functional studies on foods should include effects on the gut microbiota to provide comprehensive insight into function.

In this study, we also found that camel milk can increase the abundance of Bifidobacterium while reducing the abundance of Lactobacillus, which are both impor-

(a)

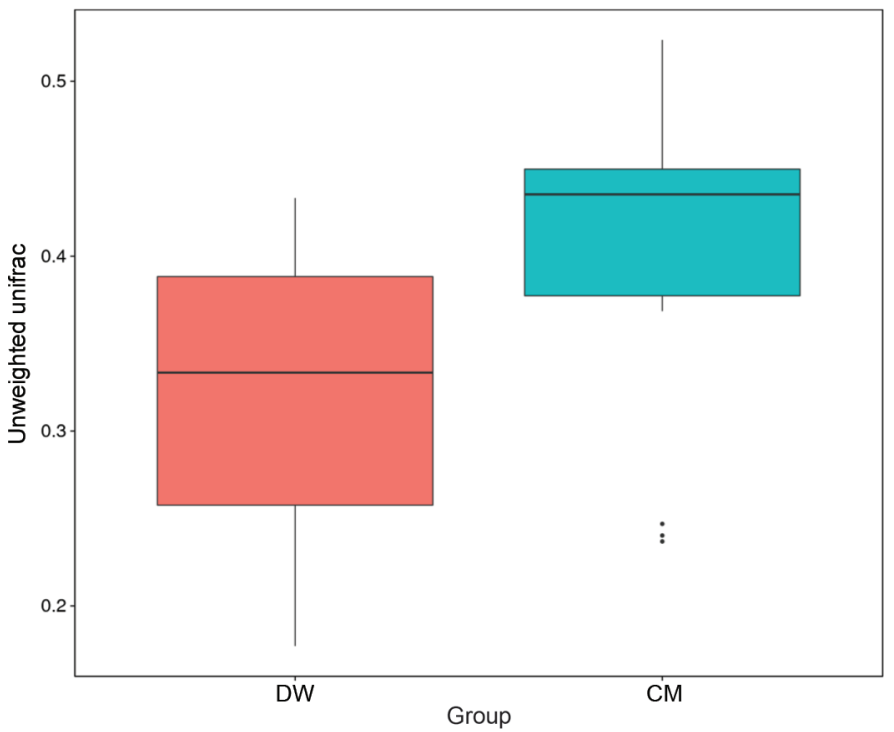

(b)

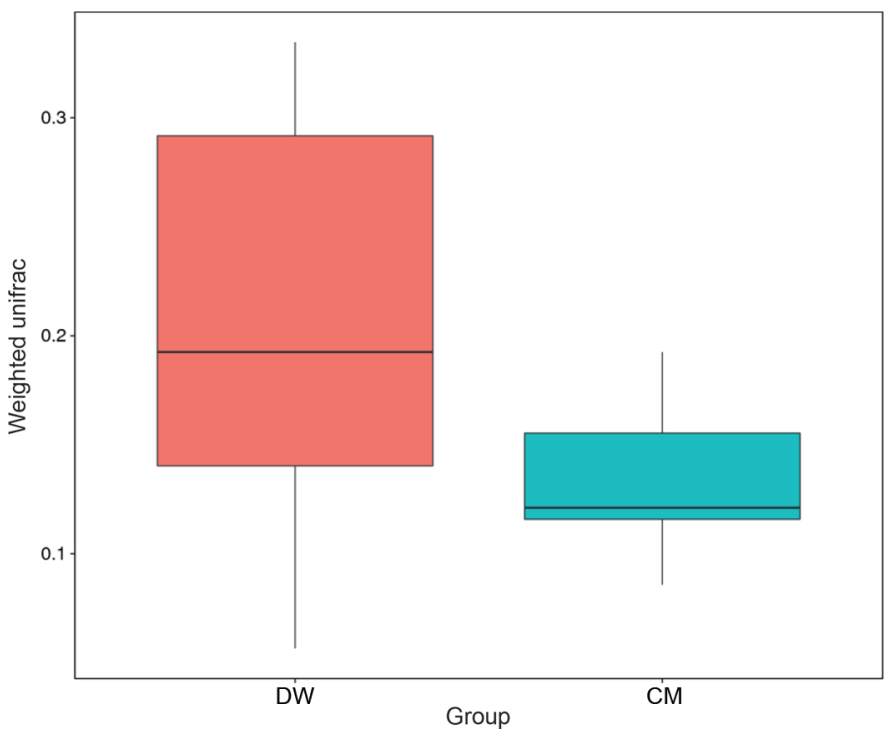

Figure 7. Beta diversity of the gut microbiota from the came milk (CM) group and the distilled water (DW) group of mice based on unweighted (a) and weighted (b) UniFrac distances. The limits of the box (the bottom and top of the box) are the first and third quartiles of the unweighted/weighted unifrac distance, and the line (band inside the box) is the second quartile of the unweighted/weighted unifrac distance (the median). The ends of the whiskers represent the minimum and maximum of the unweighted/weighted unifrac distance. The out-of-whiskers outliers are shown as dots. Color version available online. 
tant probiotics in the intestinal tract. Other genera including Turicibacter, Desulfovibrio, Pseudomonas, Lachnoclostridium, and Alistipes are also reduced following intragastric administration of camel milk, indicating that camel milk could inhibit the growth of these bacteria. Camel milk has a natural antimicrobial activity because it contains lactoferrin, lactoperoxidase, lysozyme, and immunoglobulin (Mati et al., 2017). Other antimicrobial peptides derived from camel milk have also been reported, including isracidin $\alpha_{\mathrm{S1}^{-}} \mathrm{CN}$, casecidin $\alpha_{\mathrm{S}^{-}}$and $\kappa_{-} \mathrm{CN}$, and $\beta-\mathrm{CN}$ derived peptides, which can inhibit Staphylococcus, Bacillus, Diplococcus, Streptococcus, Candida, Listeria, Klebsiella, Proteus, Pseudomonas, and Salmonella (Mohanty et al., 2016). Raw camel milk has high-level antimicrobial components (Ahamad et al., 2017), which could inhibit bacteria in the gut microbiota, leading to the lower abundance of microbes in the intestine. Regarding the increase of Bifidobacterium in the gut microbiota, there have been some reports on increased abundance of Bifidobacterium following consumption of camel milk. Half-cystine, which is similar to the insulin family of peptides and found in abundance in camel milk, could promote the growth of bifidobacteria (Abdulrahman et al., 2016). Although the lactoferrin in camel milk has bacteriostatic activity, it can also promote the growth of Bifidobacterium (Oda et al., 2014). Casein macropeptide is a peptide released from $\kappa-\mathrm{CN}$ by gastric proteinase, which can bind Streptococcus, Porphyromonas gingivalis, and Escherichia coli enterotoxin, thus inhibiting their adhesion (Malkoski et al., 2001). However, casein macropeptide could aid the proliferation of Bifidobacterium, including the species Bifidobacterium bifidum, breve, infantis, and lactis (ThomäWorringer et al., 2006). It was reported that $\alpha$-LA and glycomacropeptide from camel milk could inhibit gastrointestinal bacterial infections such as E. coli and Salmonella while improving populations of Bifidobacterium (Beermann and Hartung, 2013). Positively charged AA in the peptide could interact with the anionic compounds on certain bacterial surfaces, leading to the cell lysis (Mati et al., 2017).

Alongside the commercialization of camel milk, consumption has become more widespread and not limited

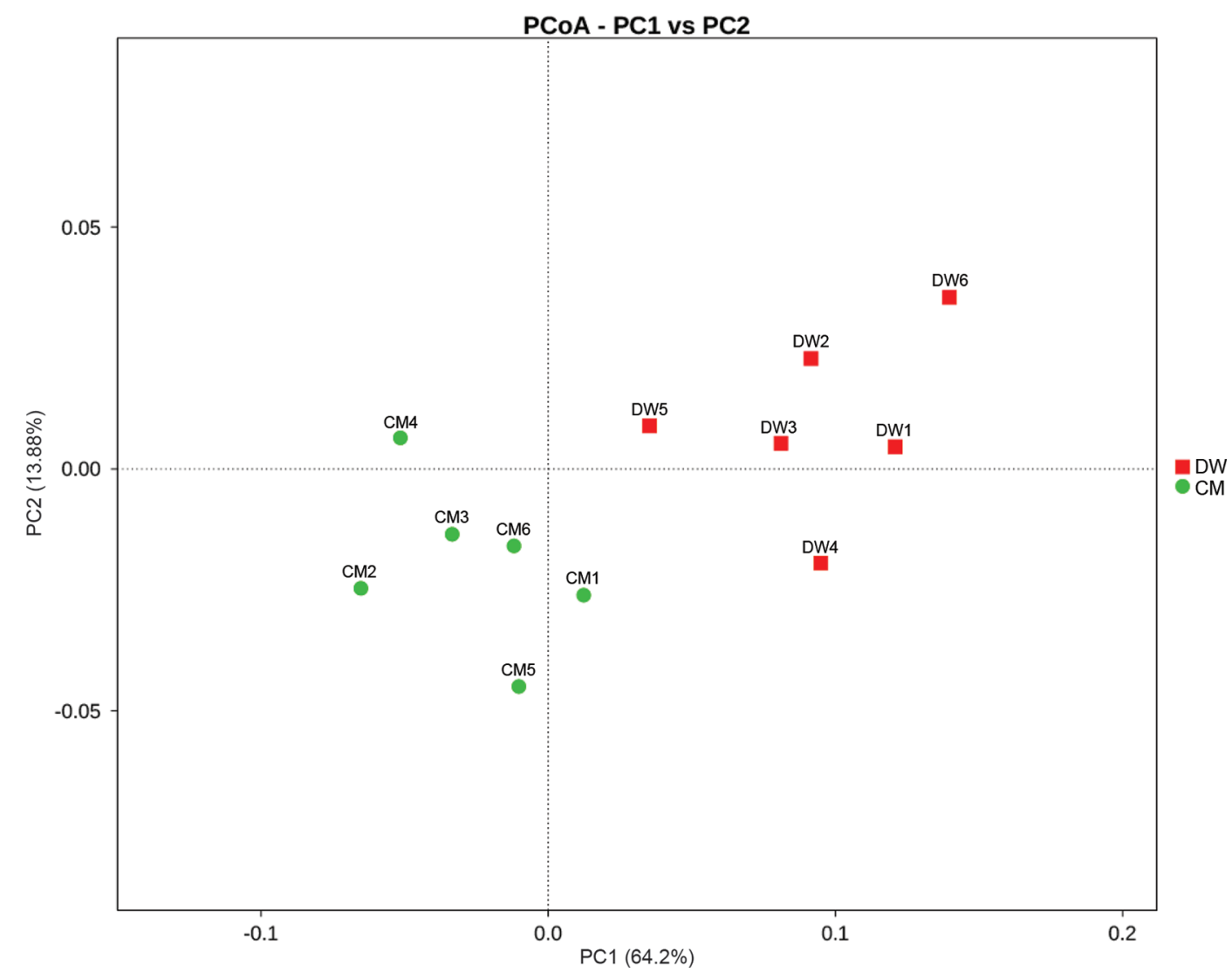

Figure 8. Principal coordinate (PC) analysis (PCoA) of the gut microbiota from the camel milk (CM) group and the distilled water (DW) group based on weighted UniFrac distance. Color version available online. 


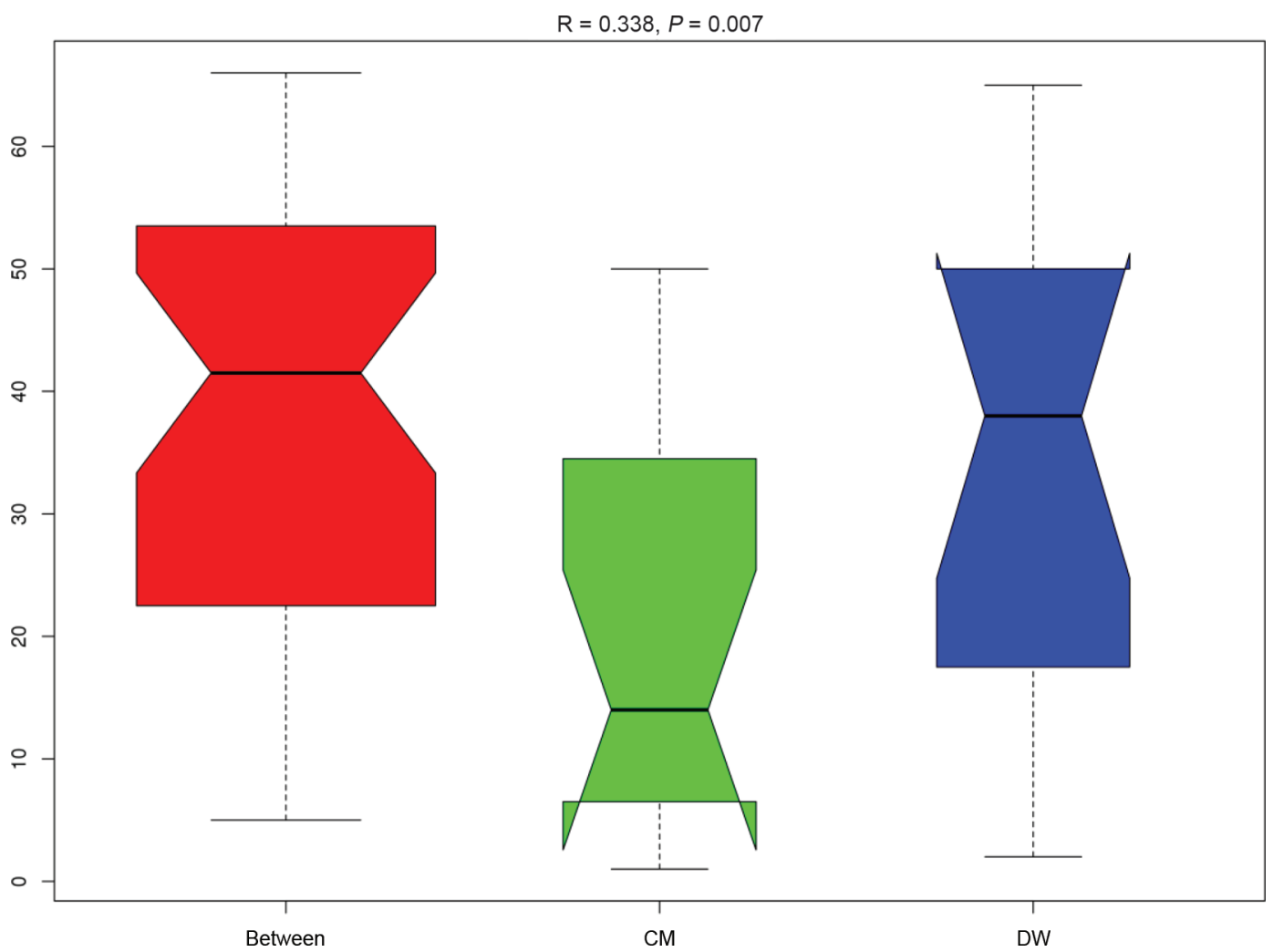

Figure 9. Anosim analysis of the difference between the gut microbiota from the camel milk (CM) group and the distilled water (DW) group of mice. The limits of the box (the bottom and top of the box) are the first and third quartiles of the rank of distance, and the line (band inside the box) is the second quartile of the rank of distance (the median). The ends of the whiskers represent the minimum and maximum of all the data within the group. Color version available online.

just to people in arid areas with access to Bactrian camel milk. Beneficial functional effects of camel milk on human health have been demonstrated. However, we propose that functional studies on camel milk should not neglect the tens of trillions of microorganisms in the gut because they also have significant effects on human health. Functional components in camel milk may interact with these gut microbiota to play a positive role in human health. In our study, we evaluated the influence of Bactrian camel milk on the composition of the gut microbiota to provide the basis for further study on the function of camel milk.

\section{ACKNOWLEDGMENTS}

This research was supported by the Fundamental Research Funds for the Central Universities (grant no. GK201603097 and GK201703063), the Major Transformation of Scientific and Technological Achievements Special Program of Shaanxi Province, China (no. 2016KTG01-02), and the Science and Technology Research and Development Program of Shaanxi Province, China (no. 2016NY-207).

\section{REFERENCES}

Abdulrahman, A. O., M. A. Ismael, K. Al-Hosaini, C. Rame, A. M. Al-Senaidy, J. Dupont, and M. A. Ayoub. 2016. Differential effects of camel milk on insulin receptor signaling-Toward understanding the insulin-like properties of camel milk. Front. Endocrinol. (Lausanne) 7:4.

Ahamad, S. R., M. Raish, A. Ahmad, and F. Shakeel. 2017. Potential health benefits and metabolomics of camel milk by GC-MS and ICP-MS. Biol. Trace Elem. Res. 175:322-330.

Ai, D., R. Huang, J. Wen, C. Li, J. Zhu, and L. C. Xia. 2017. Integrated metagenomic data analysis demonstrates that a loss of diversity in oral microbiota is associated with periodontitis. BMC Genomics 18:1041.

Arab, H. H., S. A. Salama, T. M. Abdelghany, H. A. Omar, E. A. Arafa, M. M. Alrobaian, and I. A. Maghrabi. 2017. Camel milk attenuates rheumatoid arthritis via inhibition of mitogen activated protein kinase pathway. Cell. Physiol. Biochem. 43:540-552.

Baldwin, J., B. Collins, P. G. Wolf, K. Martinez, W. Shen, C. C. Chuang, W. Zhong, P. Cooney, C. Cockrell, E. Chang, H. R. Gaskins, and M. K. McIntosh. 2016. Table grape consumption reduces adiposity and markers of hepatic lipogenesis and alters gut microbiota in butter fat-fed mice. J. Nutr. Biochem. 27:123-135.

Beermann, C., and J. Hartung. 2013. Physiological properties of milk ingredients released by fermentation. Food Funct. 4:185-199.

Belzer, C., and W. M. D. Vos. 2012. Microbes inside-From diversity to function: The case of Akkermansia. ISME J. 6:1449-1458.

Brezovečki, A., M. Čagalj, Z. F. Dermit, N. Mikulec, D. B. Ljoljić, and N. Antunac. 2015. Camel milk and milk products. Mljekarstvo 65:81-90. 
Cani, P. D., H. Plovier, M. V. Hul, L. Geurts, N. M. Delzenne, C. Druart, and A. Everard. 2016. Endocannabinoids - At the crossroads between the gut microbiota and host metabolism. Nat. Rev. Endocrinol. 12:133-143.

Cui, P., R. Ji, F. Ding, D. Qi, H. Gao, H. Meng, J. Yu, S. Hu, and H. Zhang. 2007. A complete mitochondrial genome sequence of the wild two-humped camel (Camelus bactrianus ferus): An evolutionary history of camelidae. BMC Genomics 8:241.

De Nardi, R., G. Marchesini, S. Li, E. Khafipour, K. J. C. Plaizier, M. Gianesella, R. Ricci, I. Andrighetto, and S. Segato. 2016. Metagenomic analysis of rumen microbial population in dairy heifers fed a high grain diet supplemented with dicarboxylic acids or polyphenols. BMC Vet. Res. 12:29.

Dolan, K. T., and E. B. Chang. 2017. Diet, gut microbes, and the pathogenesis of inflammatory bowel diseases. Mol. Nutr. Food Res. 61. https://doi.org/10.1002/mnfr.201600129.

Erdman, S. E. 2016. Gut microbiota: Microbes offer engineering strategies to combat cancer. Nat. Rev. Gastroenterol. Hepatol. 13:125126 .

Espín, J. C. 2017. Gut microbiota, diet and health. (We and our gut microbes). Mol. Nutr. Food Res. 61:1770015.

Galloway-Peña, J. R., D. P. Smith, P. Sahasrabhojane, W. D. Wadsworth, B. M. Fellman, N. J. Ajami, E. J. Shpall, N. Daver, M. Guindani, J. F. Petrosino, D. P. Kontoyiannis, and S. A. Shelburne. 2017. Characterization of oral and gut microbiome temporal variability in hospitalized cancer patients. Genome Med. 9:21.

Grander, C., T. E. Adolph, V. Wieser, P. Lowe, L. Wrzosek, B. Gyongyosi, D. V. Ward, F. Grabherr, R. R. Gerner, A. Pfister, B. Enrich, D. Ciocan, S. Macheiner, L. Mayr, M. Drach, P. Moser, A. R. Moschen, G. Perlemuter, G. Szabo, A. M. Cassard, and H. Tilg. 2018. Recovery of ethanol-induced Akkermansia muciniphila depletion ameliorates alcoholic liver disease. Gut 67:891-901. https:// doi.org/10.1136/gutjnl-2016-313432.

Haas, B. J., D. Gevers, A. M. Earl, M. Feldgarden, D. V. Ward, G. Giannoukos, D. Ciulla, D. Tabbaa, S. K. Highlander, E. Sodergren, B. Methé, and T. Z. DeSantis. 2011. Chimeric 16S rRNA sequence formation and detection in Sanger and 454-pyrosequenced PCR amplicons. Genome Res. 21:494-504.

Ji, Y., X. Kong, H. Li, Q. Zhu, Q. Guo, and Y. Yin. 2017. Effects of dietary nutrient levels on microbial community composition and diversity in the ileal contents of pregnant Huanjiang mini-pigs. PLoS One 12:e0172086.

Kaskous, S. 2016. Importance of camel milk for human health. Emir. J. Food Agric. 28:158-163.

Knip, M., and H. Siljander. 2016. The role of the intestinal microbiota in type 1 diabetes mellitus. Nat. Rev. Endocrinol. 12:154-167.

Konuspayeva, G., B. Faye, and G. Loiseau. 2009. The composition of camel milk: A meta-analysis of the literature data. J. Food Compos. Anal. 22:95-101.

Koropatkin, N. M., E. A. Cameron, and E. C. Martens. 2012. How glycan metabolism shapes the human gut microbiota. Nat. Rev. Microbiol. 10:323-335.

Krumbeck, J. A., M. X. Maldonado-Gomez, A. E. Ramer-Tait, and R. W. Hutkins. 2016. Prebiotics and synbiotics: Dietary strategies for improving gut health. Curr. Opin. Gastroenterol. 32:110-119.

Lajnaf, R., L. Picart-Palmade, H. Attia, S. Marchesseau, and M. A. Ayadi. 2017. Foaming and adsorption behavior of bovine and camel proteins mixed layers at the air/water interface. Colloids Surf. B. Biointerfaces 151:287-294.

Malik, A., A. Al-Senaidy, E. Skrzypczak-Jankun, and J. Jankun. 2012. A study of the anti-diabetic agents of camel milk. Int. J. Mol. Med. 30:585-592.

Malkoski, M., S. G. Dashper, N. M. O'Brien-Simpson, G. H. Talbo, M. Macris, K. J. Cross, and E. C. Reynolds. 2001. Kappacin, a novel antibacterial peptide from bovine milk. Antimicrob. Agents Chemother. 45:2309-2315.

Mati, A., C. Senoussi-Ghezali, S. S. A. Zennia, D. Almi-Sebbane, H. El-Hatmi, and J. M. Girardet. 2017. Dromedary camel milk proteins, a source of peptides having biological activities-A review. Int. Dairy J. 73:25-37.

McKenzie, C., J. Tan, L. Macia, and C. R. Mackay. 2017. The nutrition-gut microbiome-physiology axis and allergic diseases. Immunol. Rev. 278:277-295.

Mihic, T., D. Rainkie, K. J. Wilby, and S. A. Pawluk. 2016. The therapeutic effects of camel milk: A systematic review of animal and human trials. J. Evid. Based Complementary Altern. Med. 21:NP110-NP126.

Miyoshi, J., and E. B. Chang. 2017. The gut microbiota and inflammatory bowel diseases. Transl. Res. 179:38-48.

Mohanty, D. P., S. Mohapatra, S. Misra, and P. S. Sahu. 2016. Milk derived bioactive peptides and their impact on human health-A review. Saudi J. Biol. Sci. 23:577-583.

Morowitz, M. J., V. D. Caro, D. Pang, J. Cummings, B. Firek, M. B. Rogers, S. Ranganathan, R. S. B. Clark, and R. K. Aneja. 2017. Dietary supplementation with nonfermentable fiber alters the gut microbiota and confers protection in murine models of sepsis. Crit. Care Med. 45:e516-e523.

Oda, H., H. Wakabayashi, K. Yamauchi, and F. Abe. 2014. Lactoferrin and bifidobacteria. Biometals 27:915-922.

Orner, R. H., and A. H. Eltinay. 2009. Changes in chemical composition of camel's raw milk during storage. Pak. J. Nutr. 8:607-610.

Quast, C., E. Pruesse, P. Yilmaz, J. Gerken, T. Schweer, P. Yarza, J. Peplies, and F. O. Glöckner. 2013. The SILVA ribosomal RNA gene database project: Improved data processing and web-based tools. Nucleic Acids Res. 41:D590-D596.

Sa, R., R. Si, L. Fu, Q. Liu, and R. Ji. 2015. Investigation on the resources of Chinese Bactrian camel. China Herbivore Sci. 35:62-65.

Shang, Q., G. Song, M. Zhang, J. Shi, C. Xu, J. Hao, G. Li, and G. Yu. 2017. Dietary fucoidan improves metabolic syndrome in association with increased Akkermansia population in the gut microbiota of high-fat diet-fed mice. J. Funct. Foods 28:138-146.

Silbermayr, K., P. Orozco-terWengel, P. Charruau, D. Enkhbileg, C. Walzer, C. Vogl, F. Schwarzenberger, P. Kaczensky, and P. A. Burger. 2010. High mitochondrial differentiation levels between wild and domestic Bactrian camels: A basis for rapid detection of maternal hybridization. Anim. Genet. 41:315-318.

Tachon, S., J. Zhou, M. Keenan, R. Martin, and M. L. Marco. 2013. The intestinal microbiota in aged mice is modulated by dietary resistant starch and correlated with improvements in host responses. FEMS Microbiol. Ecol. 83:299-309.

ThomäWorringer, C., J. Sørensen, and R. López-Fandiño. 2006. Health effects and technological features of caseinomacropeptide. Int. Dairy J. 16:1324-1333.

Yadav, A. K., R. Kumar, L. Priyadarshini, and J. Singh. 2015. Composition and medicinal properties of camel milk: A review. Asian J. Dairy Food Res. 34:83-91.

Yan, X., X. Luo, and M. Zhao. 2016. Metagenomic analysis of microbial community in uranium-contaminated soil. Appl. Microbiol. Biotechnol. 100:299-310

Zhang, F., Z. Wang, F. Lei, B. Wang, S. Jiang, Q. Peng, J. Zhang, and Y. Shao. 2017. Bacterial diversity in goats' milk from the Guanzhong area of China. J. Dairy Sci. 100:7812-7824.

Zhang, X., Y. Zhao, M. Zhang, X. Pang, J. Xu, C. Kang, M. Li, C. Zhang, Z. Zhang, Y. Zhang, X. Li, G. Ning, and L. Zhao. 2012. Structural changes of gut microbiota during berberine-mediated prevention of obesity and insulin resistance in high-fat diet-fed rats. PLoS One 7:e42529. 